\section{$\underset{\substack{\text { hommes } \\ \text { \& migrations }}}{ }$}

\section{Hommes \& migrations}

Revue française de référence sur les dynamiques

migratoires

$1324 \mid 2019$

Religion et discrimination

\title{
L'image au service de la lutte contre les discriminations
}

\section{Emmanuelle Roule}

\section{OpenEdition \\ Journals}

Édition électronique

URL : https://journals.openedition.org/hommesmigrations/8840

DOI : $10.4000 /$ hommesmigrations. 8840

ISSN : 2262-3353

Éditeur

Musée national de l'histoire de l'immigration

Édition imprimée

Date de publication : 1 janvier 2019

Pagination : 151-157

ISBN : 978-2-919040-44-5

ISSN : $1142-852 X$

\section{Référence électronique}

Emmanuelle Roule, "L'image au service de la lutte contre les discriminations », Hommes \& migrations [En ligne], 1324 | 2019, mis en ligne le 01 janvier 2019, consulté le 06 janvier 2022. URL : http:// journals.openedition.org/hommesmigrations/8840; DOI : https://doi.org/10.4000/ hommesmigrations.8840 


\section{L'image au service de la lutte contre les discriminations}

Emmanuelle Roule, graphiste et directrice artistique freelance.

Le mardi 20 mars 2017 au Palais de la Porte Dorée, différents acteurs issus d'associations et d'institutions qui s'engagent et luttent quotidiennement contre les discriminations au sein de la société française se sont réunis à l'invitation du Musée national de l'histoire de l'immigration. Ils ont pris part à une journée d'ateliers, de débats et d'échanges sur la thématique «Images et discriminations » dans le cadre de la programmation du Grand Festival et de la semaine de lutte contre le racisme et l'antisémitisme.

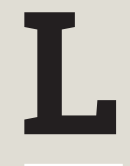

e Musée national de l'histoire de l'immigration m'a conviée à intervenir sur le Grand Festival en qualité de graphiste et de directrice artistique. Cette sollicitation portait sur la préfiguration et la mise en œuvre de l'événement, ainsi que sur le pilotage de deux ateliers professionnels à destination d'associations d'utilité publique et à dimension sociale. Portant sur la question des signes, ces ateliers avaient pour objet la production de visuels, et notamment d'affiches, pour lutter contre les discriminations au sens large.

\section{L'image comme médium}

Une image est un objet de communication agissant comme un médium qui diffuse et partage un contenu visuel et/ou textuel. L'image constitue ainsi un système de signes permettant la communication et la lecture. Quel que soit son destinataire ou le cadre du projet dans lequel sa réalisation s'insère, chaque fois que nous produisons et concevons un visuel, nous devons en préambule nous interroger sur sa destination, le cadre dans lequel il sera diffusé, et le contenu qu'il doit mettre en avant et transmettre.

Une image se manipule et se pratique. La conception d'un visuel doit considérer trois facteurs interdépendants : l'émetteur, le contexte et le récepteur. Plus largement, l'image est un message en mouvement qui a vocation à circuler. Elle vit parfois différemment pour l'émetteur et pour le récepteur car elle s'inscrit dans un contexte : elle est regardée, décryptée, interprétée et partagée. L'aspect polysémique de l'image est de ce fait une difficulté à prendre en compte lors de sa conception et de sa diffusion. 


\section{La saturation et la frénésie des images}

$\mathrm{Au}$-delà du message lui-même et de sa mise en forme visuelle, se pose également la question du cadre et des modalités de diffusion qui donneront à voir ce contenu. Nous sommes en permanence inondés d'images et de messages en tout genre, à tel point que tout individu confronté quotidiennement à ce vacarme visuel tend à la saturation. Et cette densité d'information nuit définitivement à la pertinence et à la permanence des messages.

De plus en plus, quel que soit son contenu ou son message, l'image prend une connotation négative, tant nous sommes sans cesse « agressés 》 ou « sursollicités » intellectuellement et émotionnellement par ces procédés inhérents à la communication. L'abondance nuit à la pertinence, à la circulation, à l'appropriation par le grand public du support lui-même et produit un contre-effet. Dès lors, comment exister et distinguer un message d'un autre dans un flot incessant d'accès à des contenus ? Tout designer graphique, concepteur, directeur artistique doit prendre en compte cet aspect qui implique une prise de responsabilités dans la diffusion des images.

Un autre facteur intervient également, celui de la frénésie. Si les images sont bien détentrices d'un message, elles se télescopent et se renouvellent sans permettre de les comprendre ni de se les approprier. Le temps du décryptage semble désynchronisé de celui de la diffusion. 


\section{Des ateliers de mise en pratique}

Le graphisme est une discipline qui consiste à créer, à choisir et à utiliser des éléments graphiques (dessins, typographies, photos, etc.) pour élaborer une représentation visuelle à destination d'un public. Les ateliers mis en œuvre lors du Grand Festival avaient pour intention de questionner la production d'images dans le cadre de la lutte contre les discriminations. Il s'agissait de réfléchir avec les acteurs du réseau du Musée national de l'histoire de l'immigration, à la manière de sensibiliser et de fédérer par l'image, de la façon la plus juste possible, à destination du plus grand nombre. Quelles stratégies de communication est-il possible d'utiliser en évitant tout poncif, cliché ou contresens, sans pour autant affaiblir le propos initial, tant les enjeux inhérents à la thématique de la lutte contre les discriminations sont primordiaux et d'utilité publique?

Ces deux ateliers entendaient mettre en partage et en discussion différentes thématiques et questionnements au regard des besoins de communication et des missions d'informations développées par les partenaires dans la lutte contre les discriminations, grâce à l'appui d'une expertise dans ce domaine. L'atelier est apparu comme le format le plus adapté à ce type d'échanges réunissant des groupes de travail qui peuvent ainsi facilement échanger et débattre ensemble. Les quatre principaux acteurs invités pour ce projet étaient la Ligue internationale contre le racisme et l'antisémitisme (Licra), le Centre LGBT Paris Île-de-France, Emmaüs solidarité et le Conseil général de Seine-Saint-Denis.

L'inscription aux ateliers était ouverte à tous, dans la limite de 20 places par atelier. De nombreux acteurs issus de réseaux associatifs étaient présents, mais également des étudiants en design graphique, en sociologie, en anthropologie

Les ateliers proposaient aux acteurs du Réseau du Musée un cadre d'échanges hors du temps, hors de leurs espaces de travail habituels. Durant quelques heures, leurs membres ont pu prendre du recul et consacrer du temps à un décryptage des images que leurs réalités professionnelles n'offrent que très rarement.

\section{Une thématique à caractère sensible}

Ces ateliers ont permis de débattre de la production graphique à la fois comme vecteur de mobilisation et de prise de conscience en lien avec la lutte contre les discriminations. Car cette dernière assigne à l'image un engagement qui est tout sauf neutre.

En préambule de chaque atelier, il est apparu nécessaire de se poser la question de la signification de la « discrimination». Du latin «discriminis», ce terme signifie "séparation ». Au fil des usages et du temps, il s'est imposé dans le langage courant et dans les sciences sociales avec un sens plus restreint. Dans le langage courant, la discrimination recouvre le fait de traiter de manière inégale et défavorable un ou plusieurs individus. Plus précisément, il s'agit de distinguer un groupe social des autres en fonction de caractères extrinsèques 
en France et aux États-Unis, les nombreuses polémiques que peuvent créer certaines campagnes de publicité ne relèvent pas uniquement de l'intentionnalité, mais davantage des représentations. Il ne suffit plus, pour se dédouaner, de dire qu'« on ne voulait pas être raciste». Par exemple, la marque Manix, fabricant de préservatifs, a représenté sur une affiche une femme blanche posant une main dominatrice sur la tête d'un homme noir qu'elle surplombe, avec ce slogan: "Osez être plus proches » illustre bien cette situation. "Ces représentations réductrices offrent une image tronquée de la France et de son histoire 》 dit Audrey Célestine qui poursuit: «Il est nécessaire de déconstruire ces représentations, les militants antiracistes réclament que l'histoire coloniale soit appréhendée dans sa globalité, et cela ne pourra se faire que par un travail d'éducation. » D'où une certaine lassitude qui se double de colère chez les militants associatifs et les victimes de discriminations.

1. Article 225-1 du Code pénal, Section 1 : Des discriminations, Modifié par la loi n² 2016-1547 du 18 novembre 2016 - art. 86. «Constitue une discrimination toute distinction opérée entre les personnes physiques ou entre les personnes morales sur le fondement de leur origine, de leur sexe, de leur situation de famille, de leur grossesse, de leur apparence physique, de la particulière vulnérabilité résultant de leur situation économique, apparente ou connue de son auteur, de leur patronyme, de leur lieu de résidence, de leur état de santé, de leur perte d'autonomie, de leur handicap, de leurs caractéristiques génétiques, de leurs mœurs, de leur orientation sexuelle, de leur identité de genre, de leur âge, de leurs opinions politiques, de leurs activités syndicales, de leur capacité à s'exprimer dans une langue autre que le français, de leur appartenance ou de leur non-appartenance, vraie ou supposée, à une ethnie, une nation, une prétendue race ou une religion déterminée. »

2. Voir « On peut avoir les meilleures intentions et véhiculer des représentations racistes », entretien avec Audrey Célestine réalisé par Coumba Kane, in Le Monde, 26 février 2018. 


\section{Une approche sémiotique}

Pour accompagner ce décryptage, nous avons convié aux ateliers professionnels des experts en sémiotique qui sont intervenus au fil des échanges : Anne GeslinBeyart $^{3}$ et Bernard Darras ${ }^{4}$. L'intention était de mettre à disposition des participants des outils de lecture des images et des messages, de façon scientifique, en lien avec la sémiotique, en s'affranchissant de toute subjectivité ou affect.

La sémiotique fournit les outils nécessaires à l'examen critique des symboles et des informations dans des domaines divers. Elle concerne tous les types de signes ou de symboles, et pas seulement les mots, domaine de la sémantique. Même un geste ou un son sont considérés comme des signes. Des images, des concepts, des idées ou des pensées peuvent être des symboles. Caractéristique propre à l'être humain, la faculté de manipuler des symboles lui permet d'utiliser bien mieux les relations entre les idées, les choses, les concepts et les qualités que les autres espèces vivantes.

La sémiotique est proche de la sémiologie. L'une et l'autre ont pour objet l'étude des signes et des systèmes de signification. La sémiologie renvoie davantage à Ferdinand de Saussure (son père fondateur), à Roland Barthes, à Christian Metz et, de façon plus générale, à la tradition européenne, où les sciences dites «humaines 》 restent attachées aux mouvements littéraires, esthétiques et philosophiques. La sémiotique renvoie à Charles Sanders Peirce, à Charles Morris et, plus généralement, à une tradition anglo-saxonne davantage axée sur la logique.

En amont de la journée du 20 mars 2018, j'avais souhaité que les deux sémioticiens aient accès à une sélection d'images fournies par les différents acteurs invités, en choisissant aussi bien des campagnes et des visuels qui avaient bien fonctionné, que d'autres qui avaient reçu un accueil plus mitigé, voire négatif. C'était une façon d'élaborer une approche se basant tant sur le principe de l'exemple que du contre-exemple, et d'avoir ainsi la possibilité d'échanger avec les autres interlocuteurs sur des contenus déjà diffusés.

\section{Image/action}

La journée du 20 mars s'est organisée en trois temps. Le premier, en groupe dans la matinée, a permis à tous les interlocuteurs de se rencontrer et d'échanger de façon informelle. En début d'après-midi, le groupe s'est scindé et réparti en deux espaces de travail distincts selon le principe suivant: deux acteurs associatifs en dialogue avec un sémioticien débattent et partagent leurs réflexions avec les participants.

3. Anne Geslin-Beyart, docteure en sciences du langage, spécialisée en sémiotique du design, professeure de sémiotique à l'université Bordeaux-Montaigne, directrice-adjointe du Mica (Médiation, communication, information, art), laboratoire de recherche de l'université Bordeaux-Montaigne.

4. Bernard Darras, spécialisé dans la sémiotique de la culture visuelle, matérielle et numérique et design, professeur de sémiotique et de méthodologies de la recherche à l'université Paris 1 Panthéon-Sorbonne, directeur exécutif de l'Institut Acte (UMR 8218) (Arts-créations-théories-esthétiques). 
Les deux ateliers se sont déroulés simultanément et selon des mêmes modalités : une première partie d'introduction à la sémiotique (afin de présenter cette pratique), basée sur une projection d'images issues des champs de l'art, des médias ou de campagnes de communication. Les personnes présentes étaient invitées à faire part de leurs interprétations qui étaient ensuite analysées en groupe. Alternant des quiz et des exercices pratiques, le groupe débattait autour de la signification des signes, au regard notamment des interprétations culturelles et du poids de l'affect qui conduisent à des lectures nuancées, voire différentes.

Les deux sémioticiens ont proposé des exercices pratiques d'interprétation visuelle afin de lire les images. Anne Geslin-Beyart s'est principalement axée sur la question du portrait, de son évolution au cours de l'histoire de l'art, de la peinture à la photographie, jusqu'à la question du selfie dans nos sociétés contemporaines. Bernard Darras a davantage abordé la question de la polysémie et de ses conséquences: "Face à un texte, un discours ou une image, l'attention de l'utilisateur peut être mobilisée par différents aspects du "message" et son attention peut être différente de celle de ses voisins. Il n'est donc pas étonnant de constater que devant un même fait, une même situation, un même texte, une même image, différents usagers peuvent produire des interprétations et des significations très différentes et en conséquence des réactions et actions très différentes. La polysémie est donc un phénomène normal de la communication entre les humains et ceci quels que soient le support et le média utilisés. »

Ces discussions ont amené à questionner le terme de «stéréotype ». Comme l'a expliqué Bernard Darras dans son atelier, " les stéréotypes existent de fait, dans le sens où il est normal de décrire une personne selon ses attraits physiques, toutefois ce qui fait que la notion de stéréotype bascule et devient nocive, c'est le fait d'attribuer à cette description une connotation de comportements, d'attitudes, $d^{\prime} Y$ ajouter des jugements, des interprétations majoritairement négatifs ». Ces débats ont aussi contribué à élargir le propos, notamment via l'usage de contreexemples ou de questions/tests, en essayant de comprendre dans quel contexte et à quelle époque les images décryptées avaient été conçues et diffusées.

Ces deux ateliers ont été suivis d'une restitution publique portant sur l'ensemble des événements qui avaient eu lieu au sein du musée dans le cadre du Grand Festival, en particulier sur les ateliers de production de signes graphiques menés par l'atelier Fabrication maison. Une occasion d'ouvrir un échange sur le thème «Images et dialogue citoyen ». Dans l'auditoire, nombre d'étudiants, de personnes issues d'associations militantes ont souhaité réagir, témoigner. Parfois, la prise de recul nécessaire à laquelle invitaient les deux ateliers, s'est trouvée quelque peu bousculée dans l'agora collective, où l'approche didactique et analytique s'opposait à l'emballement des émotions.

\section{Quelle suite?}

L'objectif de ces deux ateliers professionnels avec le Musée national de l'histoire de l'immigration a été véritablement de générer un cadre de discussion qui convie des intentions communes à débattre de causes nationales, avec la 


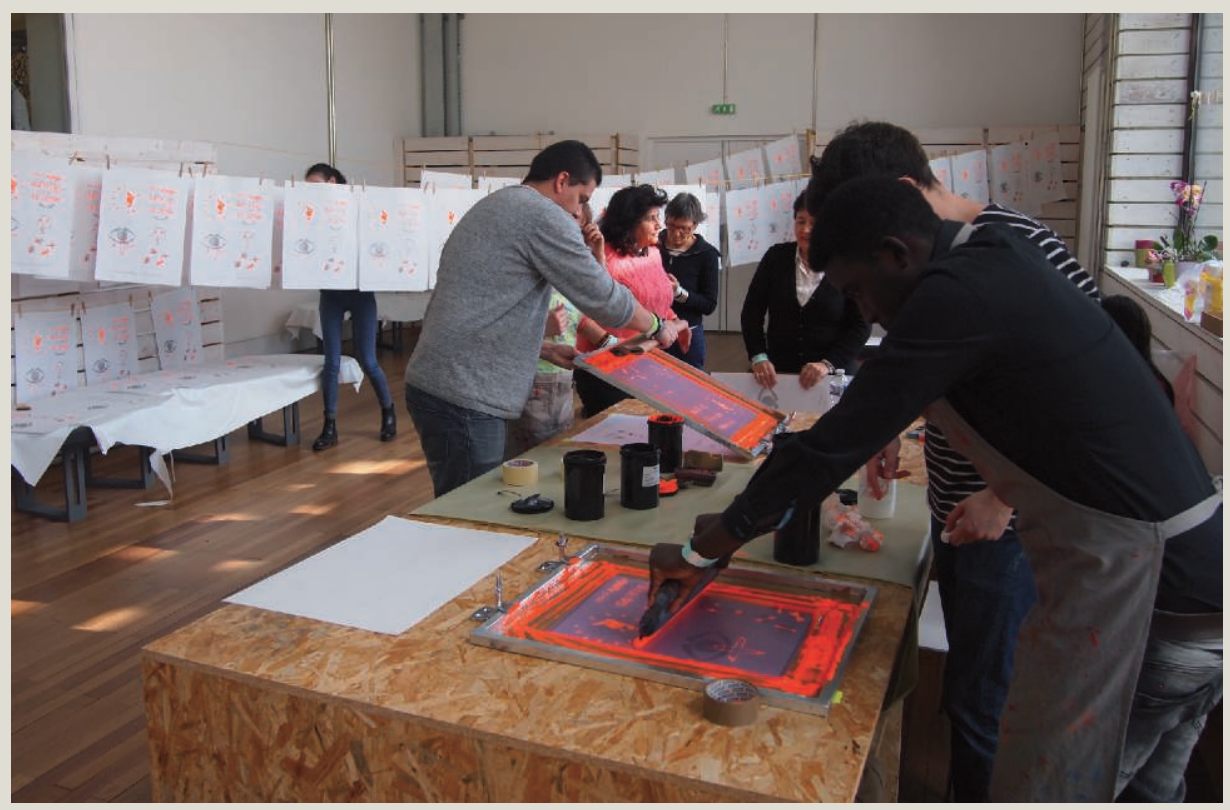

- L'atelier de sérigraphie Fabrication maison au MNHI, le 20 mars 2018, dans le cadre du Grand Festival. (C) Anne Volery.

volonté forte de déconstruire des représentations datées, plus structurelles qu'intentionnelles.

Ces deux ateliers ont permis de poser les fondements de débats utiles, partagés par l'ensemble des interlocuteurs présents. Tous souhaitent poursuivre ce qui a été amorcé conjointement, en ajustant certains éléments pour faire évoluer le format. Nombre d'entre eux ont pointé un temps d'échange trop court et souhaité différents ateliers se déroulant sur plusieurs jours.

Parmi les hypothèses envisagées afin de poursuivre ce format d'ateliers-restitution publique, une seconde édition pourrait conserver l'axe principal d'analyse des images selon le prisme de la sémiotique, en y ajoutant une deuxième expertise, celle de la sémantique. Car, au-delà de l'image, la bataille se joue aussi sur le terrain des mots, autour de leur poids, de leur impact et de leur permanence. Il serait également souhaitable de convier davantage d'étudiants en établissant en amont un travail avec des universités.

Il relève de notre rôle de citoyen et d'acteur de la société civile de penser les procédés, les modalités et les vecteurs qui permettront de dépasser les peurs et les colères liées à l'inconnu. La plupart des postures et des actes discriminatoires ne naissent que du jugement hâtif et catégorique de ce que nous ne connaissons pas. Seule l'éducation à l'image, la prise de recul, la curiosité de l'autre et sa valorisation permettront de freiner la montée des peurs, de l'obscurantisme et du repli sur soi, qui nous éloignent des autres et de nous-mêmes également. Les images n'ont pas le pouvoir de tout résoudre, mais elles ont un rôle déterminant à jouer dans la lutte contre les discriminations. La suite est à venir en 2019 . 